Pediatrics

\title{
Changes in weight and height among Chinese preschool children during COVID-19 school closures
}

\author{
Juan Wen (iD ${ }^{1,2}$, Lijun Zhu $\mathbb{D}^{1,2}$ and Chenbo Ji (D) ${ }^{1 凶}$ \\ (c) The Author(s), under exclusive licence to Springer Nature Limited 2021
}

BACKGROUND AND OBJECTIVES: COVID-19-related school closures may increase the prevalence of childhood obesity, which has aroused public concerns. We aimed to analyze the weight and height changes in Chinese preschool children during the COVID-19related school closures period.

METHODS: A total of 124,603 children from multi-city kindergartens in China were included in this study. We evaluated the prevalence of overweight and obese in preschool children experienced school closures, and compared the changes in BMI, weight, and height of preschool children among COVID-19 school closures period, the same period last year and the same period the year before last.

RESULTS: After the school closures, childhood obesity prevalence increased, whereas overweight prevalence decreased. During school closures, the average increase in height was about $1 \mathrm{~cm}$ less as compared with the same period last year and the year before last, but no noteworthy difference in the weight change was observed among the three periods.

CONCLUSIONS: During COVID-19 school closures, children's height increase seemed to be more affected than weight change. Innovative, robust, and highly adaptable strategies should be taken to increase physical activity, reduce sedentary time and promote healthy diets, to minimize the adverse impact of school closures.

International Journal of Obesity (2021) 45:2269-2273; https://doi.org/10.1038/s41366-021-00912-4

\section{INTRODUCTION}

The global spread of coronavirus disease 2019 (COVID-19) has causing substantial morbidity and mortality, and triggering a range of public health responses [1]. Following the outbreak of COVID-19 in China in January 2020, the Chinese government issued a stay-at-home order to promote social distancing [2]. Closely related to the stay-at-home orders, school closures are some of the highest-profile social distancing measures widely adopted across all of China's provinces. In addition to China, many countries around the world have also instituted a nationwide school closure [3]. These closures prevent contact among children and reduce cases [3]. In China, with the effective control of the epidemic, most schools were gradually reopened from August to September 2020. However, in most areas outside China, school closures are likely to continue through the 2020-2021 academic year. Whether and on what scale schools reopen will depend on how the epidemic evolves.

As a result of school closures, children's daily life is also affected, including insufficient physical activity, excessive sedentary behavior, and unbalanced diets [4]. It has been hypothesized that COVID-19-related school closures may expose children to an increased risk of obesity in a similar way to summer recess [5-8]. Obesity was one of the most serious global problems defined as pandemic before COVID-19 pandemic [9]. And there is a consensus that obesity is associated with various acute or chronic diseases, and also increases risk of COVID-19 infection and complications $[10,11]$. Experts in child growth have been very concerned about the potential weight and height change among preschool children during school closures. However, there is limited evidence to evaluate the impact of COVID-19related school closures on childhood obesity. Therefore, the objective of this study was to show the changes in BMI, weight, and height among Chinese preschool children during COVID-19 school closures, which could provide valuable information on designing and implementing countermeasures to minimize the detrimental impact of COVID-19 and prevent children from obesity onset.

\section{METHODS}

This was an observational retrospective study. The study participants were preschool children from kindergartens in ten Chinese cities (cities including Nanjing, Nantong, Wuxi, Chengdu, Jinan, Jiaxing, Ningbo, Qingdao, Chongqing, and Zhoushan). Children's health information of these kindergartens is managed by a Children's Health Care Network System. In this study, the examined kindergartens were selected in a cluster random sampling. All research data were based on routine physical examinations in kindergarten and extracted through the network system. Through the physical examinations, demographic information was

\footnotetext{
${ }^{1}$ Nanjing Maternity and Child Health Care Institute, Women's Hospital of Nanjing Medical University (Nanjing Maternity and Child Health Care Hospital), Nanjing, China. ${ }^{2}$ These
} authors contributed equally: Juan Wen, Lijun Zhu. ${ }^{凶}$ email: chenboji@njmu.edu.cn 

西 (n) (n)

collected, including home domicile, gender, and age. Moreover, we also collected height and weight before (December 2019 to early January 2020) and after (August 2020 to September 2020) school closures. In China, before the outbreak of the epidemic in early January 2020, kindergartens began their winter vacation and closed their schools successively. We collected baseline data of children from December 2019 to early January 2020 as the data of "before school closures". With the remission of the epidemic, kindergartens began to resume school from August to September 2020. Therefore, we collected the second data from August to September 2020 as the data of "after school closures". Weight was measured with the participant wearing light clothes and no shoes to the nearest $0.1 \mathrm{~kg}$ by a mechanical column scale, and height was measured to the nearest $0.1 \mathrm{~cm}$ with a Stadiometer. Each measurement was taken and recorded twice to ensure reliability. From these data, we calculated body mass index (BMI), and the changes in BMI, weight, and height from before until after school closures. Missing data included data for children who did not attend the physical examination before or after school closures, or abnormal value (during the COVID-19 school closures, weight change exceed $\pm 15 \mathrm{~kg}$ or height change exceed $15 \mathrm{~cm}$ ).

We applied Fisher's exact test to evaluate the difference in the prevalence of overweight and obese children before and after school closures. Overweight and obesity were diagnosed according to the child growth standards of the WHO [12]. To further vertically explore the impact of COVID-19 school closures on obesity among preschool children, we then compared the changes in BMl, weight, and height of preschool children among the COVID-19 school closures period, the same period last year and the same period the year before last. The participant sources, time points and methods of data collection, and exclusion criteria for the same period last year and the year before were the same as those during the COVID-19 school closures. The changes in BMI, weight, and height are graphically plotted as mean in the figures. Statistical data were analyzed by IBM SPSS version 25 . The study did not require approval by the ethics committee because of the anonymous nature of the personal information and impossibility of tracking sensitive personal data.

\section{RESULTS}

In the past year under COVID-19 pandemics, 19,066 preschool children who attended routine kindergarten physical examination before and after school closures were included in the analysis (Table 1). The children evaluated were 3 years to 5 years and 4 months during the months before school closures (December 2019 to early January 2020). Using the recorded height and weight together with children's sex and age, we observed the overweight rate had decreased (before vs. after school closures: $17.43 \%$ vs. $14.07 \%, P<0.001)$, whereas the obesity rate had increased (before vs. after school closures: $10.47 \%$ vs. $12.28 \%, P<0.001$ ) during the COVID-19-related school closures.

Using a retrospective cohort design, we compared changes in anthropometrics of preschool children among COVID-19 school closures period, the same period last year and the same period the year before last. Table 1 shows the basic characteristics of participating children evaluated in these three periods. Given that participants from the three periods came from same cities, had similar population structure, and the baseline anthropometrics were very close, the previous data were of certain reference value for assessing the impact of COVID-19 on obesity.

We first compared changes in BMI over the three studied periods, as shown in Fig. 1. It is well known that BMI decreases with age between 6 months and 6 years old [12]. During school closures, BMI decreased the least when compared with the same period last year and the year before last. In addition, there was a small increase in BMI rather than a decrease among the relatively older children. To investigate the reasons behind the changes in BMI, we analyzed the changes in weight (Fig. 2). However, no noteworthy difference in the weight change was observed among the three periods. When we further analyzed the changes in children's height (Fig. 3), we were surprised to find that the average increase in height during school closures was about 


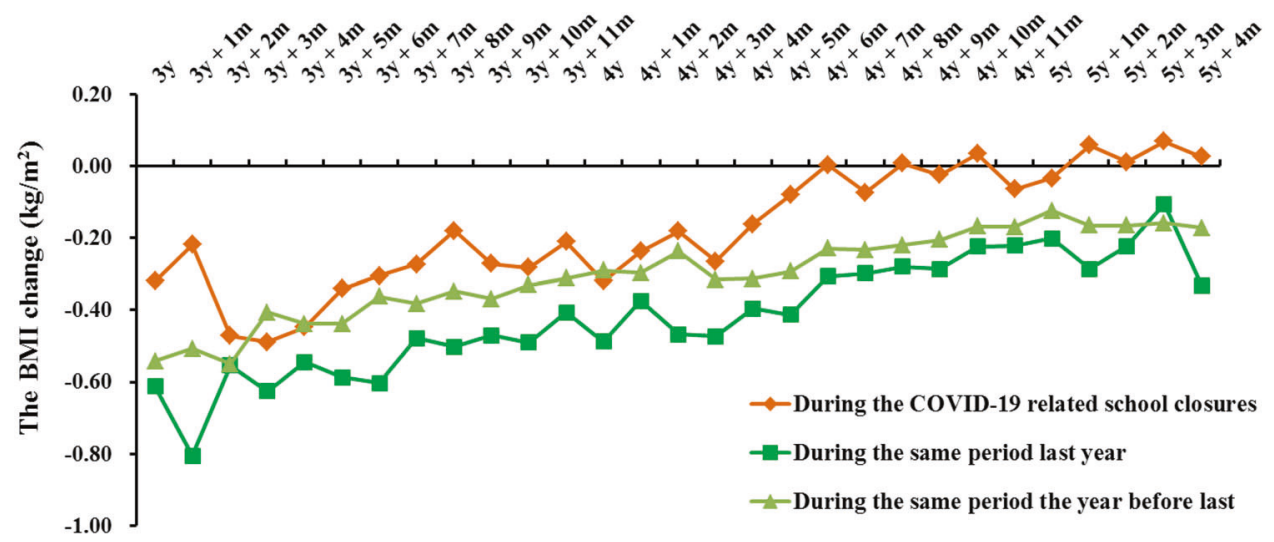

Fig. 1 The BMI change of the preschool children over the three periods. Compared to the pre-COVID-19 periods, preschool children's BMI decreased the least during the school closures.
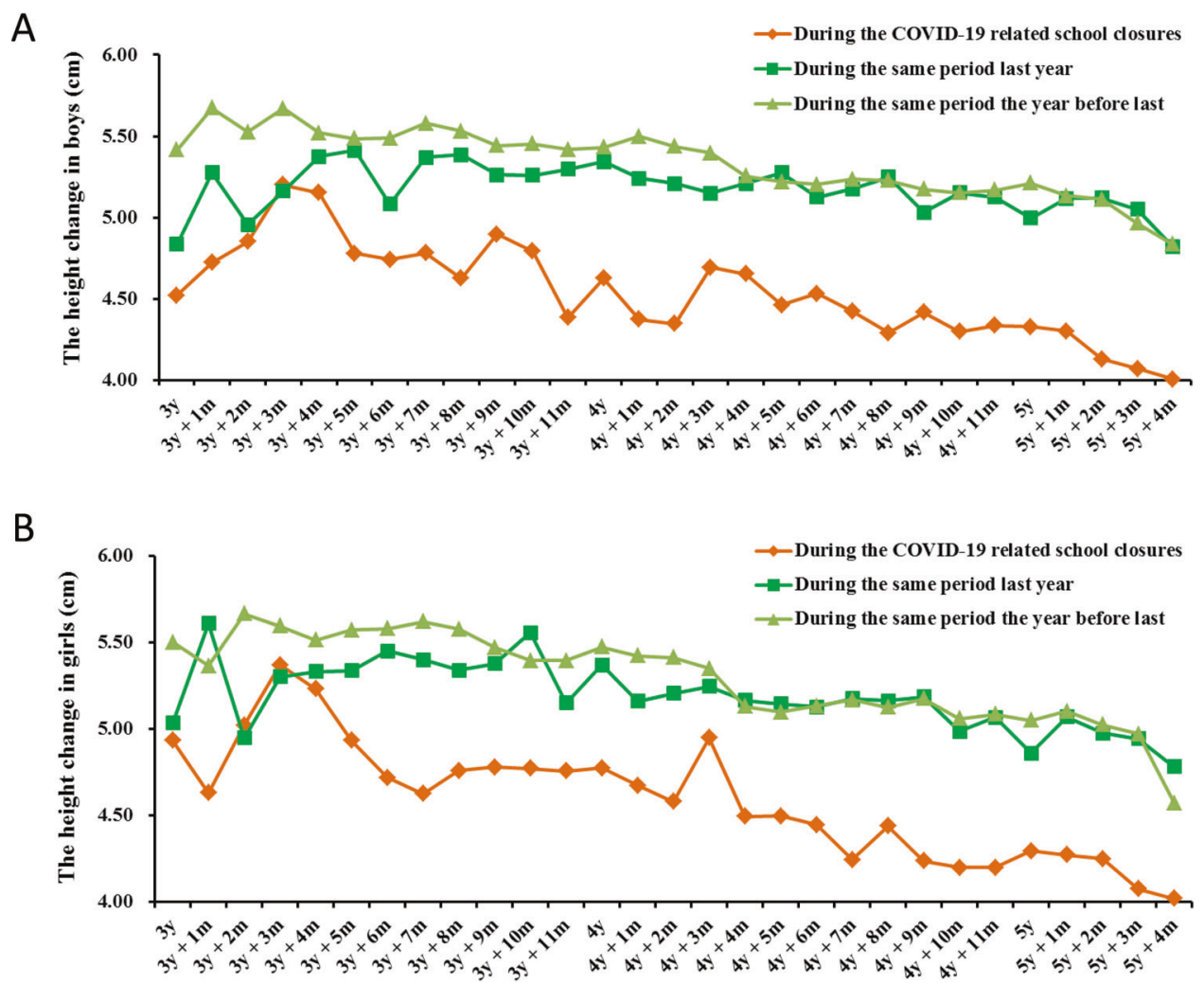

Fig. 2 The height change of the preschool children over the three periods. A The height change in boys; B The height change in girls.

$1 \mathrm{~cm}$ less, compared with the same period last year and the year before last. This may be partly responsible for the increased obesity rate and the least BMI decrease during school closures. However, according to the child height standard of the WHO [12], for the preschool children evaluated during school closures, $71.4 \%$ of the children had a height value above P50 and $14.6 \%$ have a height value above P95 after school closures, indicating that the preschool children group is still in a good growth state.

\section{DISCUSSION}

The currently escalating COVID-19 pandemic represents one of the most profound societal and public health challenges. Although school closures were necessary to slow the spread of COVID-19, they may have a profound impact on children's daily life. As suggested by growing evidences, unfavorable changes in eating, sleep, and activity behaviors occurred during the school closures
[13-15]. Most obviously children typically engage in lower levels of physical activity and more sedentary time as compared to school days [13]. And many children have experienced unbalanced diets during the pandemic response [4]. In sum, the COVID19 school closures have reinforced the obesogenic environment. As expected, our results showed that childhood obesity prevalence increased after the COVID-19-related school closures, and compared to the pre-COVID-19 periods, preschool children's BMI decreased the least during the school closures.

There is considerable concern about whether the rising obesity rate is related to unhealthy weight gain. Although recent studies have concluded that the reduced physical activity resulting from the enforced quarantine would contribute to weight gain $[13,16,17]$, we observed no noteworthy difference in the children's weight change among the three studied periods. More surprisingly, there was even a drop in the children overweight rate after the school closures period. During the school closures, in 
A

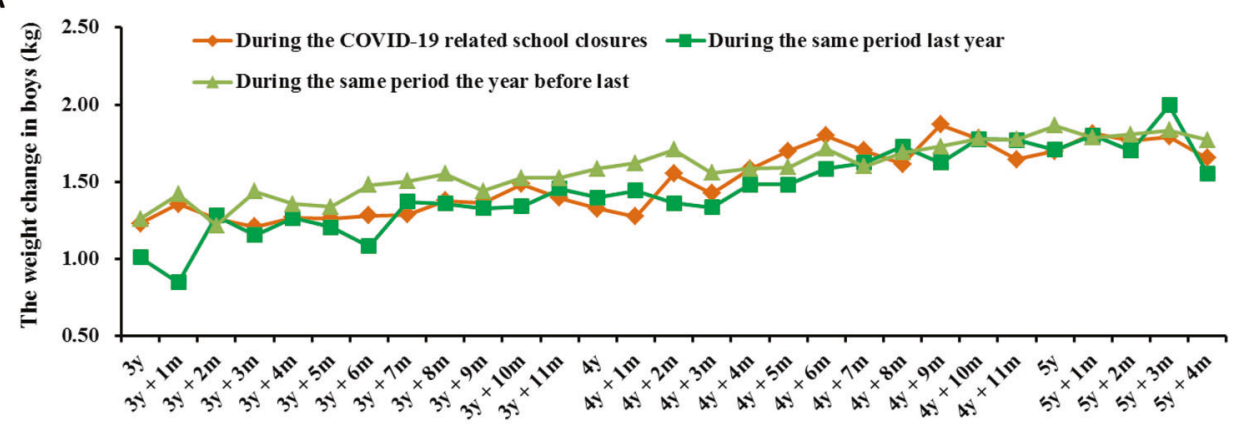

B

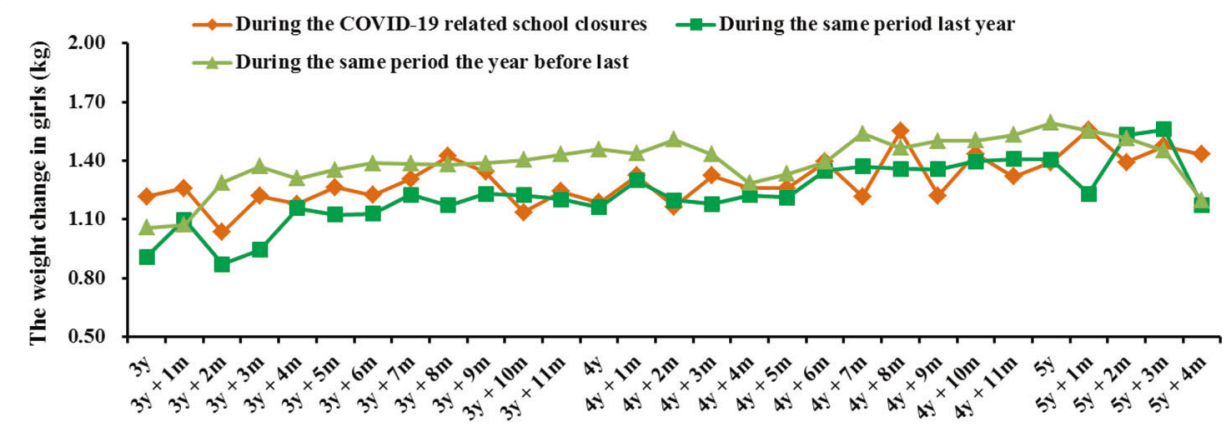

Fig. 3 The weight change of the preschool children over the three periods. A The weight change in boys; B The weight change in girls.

response to social distancing policies, children may eat less frequently in restaurants, usually rich in fats, sugars, and salt. Experts in sports medicine have called for that diet matters more than exercise in weight loss, and poor diet generates more disease than physical inactivity, alcohol, and smoking combined [18]. Based on our results, we thus consider that, during the school closures, the effect of weight loss due to reduced calorie intake was more powerful than weight gain due to reduced physical activity. Furthermore, it was suggested that over a long period of time children who are overweight may lower body weight relative to changes in height [19], which also supported our results. In addition, the decreased overweight rate may partly contribute to the increased obesity rate, as a proportion of children who were overweight became obese.

For height changes, compared with the pre-COVID-19 periods, the average increase in height during school closures was about $1 \mathrm{~cm}$ less, suggesting the reduced physical activity due to school closures may have contributed to the limited growth in height. The latest statistics show that over the past 35 years (from 1985-2019), China has become the country with the largest gains in height for children and teenagers aged 5-19 [20]. And after school closures, we evaluated the preschool children's height, and found they were still in a good growth state with most children had a height value above P50. Even so, if COVID-19 school closures lasting a year or more, there may be enormous consequences for children's overall physical health. In order to minimize the adverse impact of COVID-19, innovative, robust, and highly adaptable strategies must be taken to promote physical activity at home or in residential settings. And schools could make concerted efforts to incorporate physical activities into distance learning curriculum. Moreover, a pro-healthy diet based on rich protein-low fat food, healthy fats, and plant food is also a key strategy.

To the best of our knowledge, this was the first study analyzing weight and height changes in preschool children during the COVID-19-related school closures period. The strengths of the study included the timeliness of the baseline survey across the school closures period and wide geographic representation. Yet, some limitations existed. First, our sample was not geographically equally distributed, with almost half of the participants resided in Jiangsu province (relatively developed areas) and none in Hubei province (outbreak site of COVID-19). Therefore, the observed impact of the pandemic on childhood obesity may have been diminished. Second, this study concerns children in kindergarten years so that the findings may not be generalizable to all children and adolescents. Lastly, the lack of information on diet and physical activity may limit the comparability of outcomes between the COVID-19 period and pre-COVID-19 periods studied. Further studies in larger, more racially/ethnically diverse populations are needed to confirm the preliminary results.

Overall, height increase in children seemed to be more affected than weight change during the school closures. Of public health concern is these short-term changes in response to COVID-19 may become permanent, leading to an increased risk of obesity in children as they get older. Programmatic and policy strategies during the pandemic should therefore focus on increasing physical activity, reducing sedentary time, and promoting healthy diets, which deserves full support and dissemination by all of us. In a comprehensive evaluation of the effective control of the epidemic and the slight impact on children's height, school closure is more beneficial than harmful in the short term.

\section{REFERENCES}

1. Helmy YA, Fawzy M, Elaswad A, Sobieh A, Kenney SP, Shehata AA. The COVID-19 pandemic: a comprehensive review of taxonomy, genetics, epidemiology, diagnosis, treatment, and control. J Clin Med. 2020;9:1225.

2. Leung K, Wu JT, Liu D, Leung GM. First-wave COVID-19 transmissibility and severity in China outside Hubei after control measures, and second-wave scenario planning: a modelling impact assessment. Lancet. 2020;395:1382-93.

3. Bayham J, Fenichel EP. Impact of school closures for COVID-19 on the US healthcare workforce and net mortality: a modelling study. Lancet Public Health. 2020;5: e271-e8. 
4. Rundle AG, Park Y, Herbstman JB, Kinsey EW, Wang YC. COVID-19-related school closings and risk of weight gain among children. Obesity. 2020;28:1008-9.

5. Clemmensen C, Petersen MB, Sorensen TIA. Will the COVID-19 pandemic worsen the obesity epidemic? Nat Rev Endocrinol. 2020;16:469-70.

6. Workman J. How much may COVID-19 school closures increase childhood obesity? Obesity. 2020;28:1787.

7. An R. Projecting the impact of the coronavirus disease-2019 pandemic on childhood obesity in the United States: a microsimulation model. J Sport Health Sci. 2020;9:302-12.

8. von Hippel PT, Workman J. From kindergarten through second grade, U.S. children's obesity prevalence grows only during summer vacations. Obesity. 2016;24:2296-300.

9. Meldrum DR, Morris MA, Gambone JC. Obesity pandemic: causes, consequences, and solutions-but do we have the will? Fertil Steril. 2017;107:833-9.

10. Kumar S, Kelly AS. Review of childhood obesity: from epidemiology, etiology, and comorbidities to clinical assessment and treatment. Mayo Clin Proc. 2017:92:251-65.

11. Demeulemeester F, de Punder K, van Heijningen $M$, van Doesburg F. Obesity as a risk factor for severe COVID-19 and complications: a review. Cells. 2021;10:933.

12. Group WHOMGRS. WHO child growth standards based on length/height, weight and age. Acta Paediatr Suppl. 2006;450:76-85.

13. Dunton GF, Do B, Wang SD. Early effects of the COVID-19 pandemic on physical activity and sedentary behavior in children living in the U.S. BMC Public Health. 2020;20:1351.

14. Pietrobelli A, Pecoraro L, Ferruzzi A, Heo M, Faith M, Zoller T, et al. Effects of COVID-19 lockdown on lifestyle behaviors in children with obesity living in Verona, Italy: a longitudinal study. Obesity. 2020;28:1382-5.

15. Ntambara J, Chu M. The risk to child nutrition during and after COVID-19 pandemic: what to expect and how to respond. Public Health Nutr. 2021;1-7. Online ahead of print.

16. He M, Xian Y, Lv X, He J, Ren Y Changes in Body Weight, physical activity, and lifestyle during the semi-lockdown period after the outbreak of COVID-19 in China: an online survey. Disaster Med Public Health Prep. 2020;1-6. Online ahead of print.

17. Jia P, Zhang L, Yu W, Yu B, Liu M, Zhang D, et al. Impact of COVID-19 lockdown on activity patterns and weight status among youths in China: the COVID-19 Impact on Lifestyle Change Survey (COINLICS). Int J Obes. 2021;45:695-9.

18. Malhotra A, Noakes T, Phinney $\mathrm{S}$. It is time to bust the myth of physical inactivity and obesity: you cannot outrun a bad diet. Br J Sports Med. 2015;49:967-8.
19. Williamson DA, Han H, Johnson WD, Stewart TM, Harsha DW. Longitudinal study of body weight changes in children: who is gaining and who is losing weight. Obesity. 2011;19:667-70.

20. Collaboration NCDRF. Height and body-mass index trajectories of school-aged children and adolescents from 1985 to 2019 in 200 countries and territories: a pooled analysis of 2181 population-based studies with 65 million participants. Lancet. 2020;396:1511-24.

\section{AUTHOR CONTRIBUTIONS}

JW conceived and designed the idea, wrote, and drafted the manuscript. LZ did data collection and performed the data analysis. CJ designed, contributed to the reviewing of the final manuscript. All authors approved the final format of the submitted manuscript.

\section{FUNDING}

This work was supported in part by the National Natural Science Foundation of China (81770866, 81600685), Jiangsu provincial key research and development program (BE2020626), 333 high level talents training project of Jiangsu Province, and Six talent peaks project in Jiangsu Province (YY-081, WSN-121)

\section{COMPETING INTERESTS}

The authors declare no competing interests.

\section{ADDITIONAL INFORMATION}

Correspondence and requests for materials should be addressed to C.J.

Reprints and permission information is available at http://www.nature.com/ reprints

Publisher's note Springer Nature remains neutral with regard to jurisdictional claims in published maps and institutional affiliations. 